\title{
1. Simulation of Flow around Rigid Vegetation 2 Stems with A Fast Method of High Accuracy
}

\author{
Don Liu ${ }^{\mathrm{a}, *}$ \\ a Mathematics 83 Statistics, and Mechanical Engineering \\ Louisiana Tech University, Ruston, LA 71272 USA \\ Yonglai Zheng ${ }^{b, * *}$ \\ ${ }^{\mathrm{b}}$ Department of Hydraulic Engineering, School of Civil Engineering \\ Tongji University, Shanghai, 200092, China \\ Qin Chen ${ }^{\mathrm{c}}$ \\ ${ }^{\mathrm{c}}$ Department of Civil and Environmental Engineering \\ and Center for Computation and Technology \\ Louisiana State University, Baton Rouge, LA 70803 USA
}

\begin{abstract}
This paper develops the Virtual Cylinder Model (VCM) to simulate flows through vegetation canopies at low to medium high Reynolds numbers in wetlands. Using a combined Eulerian and Lagrangian approach, the VCM is capable of simulating the flow field around each vegetation stem (modeled as a cylinder) as well as a bulk of numerous stems in a fluid. Numerical results of flows through regularly and randomly arranged cylinders have been compared with direct numerical simulations. Good agreement has been achieved. This model maintains an excellent balance between accuracy and efficiency in modeling interactions between the fluid and vegetation stems. Simulation results demonstrate that this model is promising for investigating the hydrodynamics and vegetation resilience in wetlands that serve as a first line of defense against the sea level rise, storm impacts and coastal erosion.
\end{abstract}

Key words: Fluid-structure interaction; Navier-Stokes equations; Spectral modal element method; Direct numerical simulation

* Corresponding author: DonLiu@latech.edu; Tel.: +1 3182574670

**Second corresponding author: ZYLL@tongji.edu.cn

Preprint submitted to Elsevier Science 


\section{Introduction}

Field and laboratory studies have shown that vegetation in wetlands can effectively reduce the speed of water flow [1,2], modify turbulence structure [3,4], attenuate wave energy [5-8], and affect sediment transport [9-11]. A good review of wave energy dissipation by vegetation was given in [12]. Recent numerical simulations using the depth-integrated, nonlinear shallow water equations have demonstrated the potential of flood risk reduction provided by coastal wetlands [13-15]. Wetland restoration has been promoted for ecological benefits and flood mitigation.

Vegetation stems in coastal wetlands, such as salt marshes, have been treated as rigid cylinders of small diameters in the literature. Zdravkovich [16] conducted extensive experimental studies on flow past cylinders, from a single cylinder to cylinder arrays at different configurations and Reynolds numbers. Most work in the numerical modeling of flow through vegetation canopies uses the Reynolds-averaged NavierStokes (RANS) equations with isotropic or anisotropic turbulence models $[17,10,18$ 20]. Large eddy simulation (LES) has also been applied to fluid and vegetation interactions [21-24]. Recent numerical studies [15,25] implemented with theoretically derived drag formulations were conducted for deformable vegetation (emergent and submerged). The vegetation effects on the flow field have been parametrized as a momentum sink term in the RANS equations. However, using parametrized Direct Numerical Simulation (DNS) on such kind of flow at low to moderately high Reynolds is rare, partially because of the extremely high computational cost to resolve boundary layers of many vegetation stems at the same time.

Immersed boundary methods (IBM), first introduced by Peskin [26,27], an alternative to DNS, are quite popular in modeling fluid structure interactions using stationary Eulerian grids. In IBM simulations, nonslip boundary conditions on immersed solid surfaces are not imposed and body-conforming mesh is not used at all. Instead, interaction forces between a fluid and a solid structure are explicitly computed from solid boundary grids using the knowledge of the solid boundary, before these forces are interpolated back to nearby fluid grids. Unverdi and Tryggvason [28] numerically simulated complex multi-fluid flows. Fauci and McDonald [29] numerically studied both the rigid and elastic biological organisms immersed in water. Daiki and Biringen [30] used a feedback forcing term added to the momentum equations to simulate a single cylinder motion in a uniform flow. Mohd-Yosuf [31] extracted the forcing terms directly from numerical solutions. A detailed and insightful review was given in Mittal and Iaccarino [32].

Field measurements of tidal currents in coastal wetlands show that the flow velocity ranges $0.02 \sim 0.06 \mathrm{~m} / \mathrm{s}$ adjacent to the vegetation canopy and $0.01 \sim 0.04 \mathrm{~m} / \mathrm{s}$ within the canopy $[33,34]$. As the first step, the focus of this study is on flows with low to moderate Reynolds numbers. This represents realistic conditions under typical tidal forcing in coastal wetlands. 
The objective of this paper is to develop a computational model that is capable of resolving individual vegetation stem as well as describing the interaction between a bulk of numerous vegetation stems with a fluid, with a decent accuracy compared with DNS but at only a small fraction of the computational cost of DNS. This computational model does not resolve the boundary layers near the surface of the vegetation stems but use tailored force fields to characterize the interactions. Similar ideas have been successfully used in particulate flow simulations $[35,36]$.

Sections of this paper are organized as below. First, the current state of practice of vegetation attenuation in wetlands are introduced and the background of existing methods are reviewed. Second, the computational physics method, Virtual Cylinders Model (VCM), is explained in detail. Third, several sets of numerical experiments are presented and results are validated to verify the accuracy of VCM. Fourth, applications of VCM are demonstrated for flows past randomly arranged numerous cylinders which are very expensive to replicate in laboratories or direct numerical simulations. Simulation results are further discussed before a summary of findings and a conclusion are provided.

\section{Simulation Approaches}

To simulate the hydrodynamic interactions between water and vegetation canopies, simulated as cylinders, especially a large number of them, one way is to fully resolve the water boundary layers around all cylinders to obtain drag, lift and torque between the water and cylinders. This practically requires prohibitively complex discretization with a massive number of body-fitting elements and grid points. This paper presents an economical and accurate approach to handle this challenge. This approach uses a fixed Eulerian reference for the fluid and a penalized Lagrangian reference for each cylinder. Instead of using tiny elements around each cylinder, only virtual cylinders are modeled and the entire domain is discretized with fixed and relatively coarse elements. Therefore, this computational model is called Virtual Cylinders Model (VCM). Instead of resolving the boundary layer outside a cylinder, VCM imposes tailored force fields, which is time and space dependent for a cylinder, and resolves its interior volume and its vicinity. If this cylinder moves with water, then Lagrangian kinetic equations are integrated to compute its position and tilted angle at the next time step. Since avoiding resolving boundary layers around cylinders, this approach significantly reduces the computational cost by one to two order of magnitude in wall clock time compared to DNS. Therefore, it has the potential for modeling numerous vegetation stems. 
Because of the efficiency of the Eulerian description for the fluid, the incompressible Navier-Stokes equations in the Eulerian form with added source terms are used to describe the fluid motion. The momentum and mass conservation for the fluid in terms of the primitive variables, $\mathbf{u}$ and $p$, are

$$
\rho \frac{D \mathbf{u}}{D t}=-\nabla p+\mu \nabla^{2} \mathbf{u}+\sum_{k=1}^{N}\left(\mathbf{F}^{k}+\overrightarrow{\mathbf{G}}^{k} \nabla\right) \Phi\left[\mathbf{x}-\mathbf{Y}^{k}(t), \sigma, t\right], \quad \nabla \cdot \mathbf{u}=0
$$

where $\rho$ and $\mu$ are the density and viscosity of the fluid; $\mathbf{x}$ is the Eulerian coordinates. The last term on the right characterizes the momentum exchange between the fluid and $N$ cylinders including the cylinder $k$ located at $\mathbf{Y}^{k}(t)$. The kernel function in the last term of equation (1), $\Phi\left[\mathbf{x}-\mathbf{Y}^{k}(t), \sigma, t\right]$ is the spatial filter function. For zero to moderately high Reynolds number flows, which is defined with the diameter of a cylinder, the filter function is:

$$
\Phi\left[\mathbf{x}-\mathbf{Y}^{k}(t), \sigma, t\right]=\frac{1}{2 \pi \sigma^{2} L} \operatorname{Exp}\left[-\frac{1}{2 \sigma^{2}} \psi\left(\mathbf{x}, \mathbf{Y}^{k}(t)\right)\right]
$$

where $\psi\left(\mathbf{x}, \mathbf{Y}^{k}(t)\right)$ is the squared distance from a point at $\mathbf{x}$ to the axis of symmetry of the $k^{t h}$ cylinder with radius $r$; the parameter $\sigma=r \pi^{-\frac{1}{2}}$ is to set the range of this filter function in order to match the Stokes drag. This setting was derived and has been successfully used in Maxey et al. [37,38] and Liu et al. [39,40]. For vegetation stems with non-circular cross sections, e.g., elliptical, the filter function could be adjusted with an appropriate $\sigma$ that reflects the actual aspect radio and shape of the vegetation stem. Modeling vegetation stems of different sizes and shapes is readily applicable through adjusting the filter function.

The term $\mathbf{F}^{k}$ in equation (1) contains the forces on the fluid from the cylinder $k$. It is the vector sum of the net inertial effect due to the added mass and the counteracting forces $\mathbf{F}_{B}^{k}$ for drag (called balancing drag) along the mainstream direction and lift (called balancing lift) in the lateral direction:

$$
\mathbf{F}^{k}=\left(m_{f}-m_{c}\right) \frac{d \mathbf{V}^{k}}{d t}+\mathbf{F}_{B}^{k}
$$

where, $m_{c}$ and $m_{f}$ are the mass of the $k^{t h}$ cylinder and the mass of displaced fluid, respectively; $\mathbf{V}^{k}$ is the nominal velocity of the $k^{t h}$ cylinder. When the cylinder is fixed, $\mathbf{V}^{k}$ is obviously zero. However, for both fixed and moving cylinders, the drag, lift, and torque could be computed by penalizing the corresponding velocity of the cylinder. Therefore, the velocity is kept in the formulation. The balancing force $\mathbf{F}_{B}^{k}$ is elaborated in Section 2.2. Gravity and buoyancy forces do not appear in here as the vertical motion is negligible and the flow is mainly in the mainstream and lateral directions. 
The term $\overrightarrow{\mathbf{G}}^{k}$ in equation (1) contains the balancing torques from the cylinder to the fluid. It is a second order tensor which could be decomposed into the sum of the symmetric and antisymmetric parts. The symmetric part neutralizes the pressure difference around the cylinder and prevents the virtual cylinder from deviating away from its center of mass. The antisymmetric part contains torques in different planes exerting on the fluid due to shear stress. Both parts were computed iteratively in the dipole iteration scheme $[39,41]$, to adjust the volume-averaged strain rate tensor within the cylinder volume to mimic the zero motion interior to the solid vegetation stems. Components of $\overrightarrow{\mathbf{G}}^{k}$ help reduce the integrated strain rate tensor within the cylinder to zero. This is because there is no net flow inside a real vegetation and the integral filtered strain rate tensor within the volume of a virtual cylinder should be a zero tensor of second order:

$$
\iiint_{V o l} \overrightarrow{\boldsymbol{\epsilon}} \Phi\left[\mathbf{x}-\mathbf{Y}^{k}(t), \sigma, t\right] d V o l \approx 0
$$

where $\overrightarrow{\boldsymbol{\epsilon}}$ is the strain rate tensor; $\mathrm{Vol}$ is the volume of the computational domain.

\subsection{Balancing Drag, Lift and Torque}

Since it is unnecessary to resolve the non-slip surface boundary layer on each cylinder in the VCM formulation, tiny curvature-fitting elements are avoided in VCM runs. In order to obtain the unknown drag, lift and torque on each cylinder, the nominal velocity (translational and rotational) of the cylinder is penalized to compute the unknowns which counteract the drag, lift and torque on the cylinder. To implement this penalty algorithm, a pseudo-time $\tau$ was introduced between two consecutive time steps and a set of pseudo-time-dependent problems about the balancing force and torque were solved:

$$
\begin{gathered}
\frac{\partial \mathbf{F}_{B}^{k}(t, \tau)}{\partial \tau}=-\lambda_{1} \mathbf{V}^{k}(t, \tau), \\
\frac{\partial T_{i j}(t, \tau)}{\partial \tau}=-\lambda_{2} \epsilon_{i j k} \Omega_{k}(t, \tau),
\end{gathered}
$$

where $\mathbf{F}_{B}^{k}(t, \tau)$ is the balancing force as in equation $(3) ; T_{i j}(t, \tau)$ is the component of $\overrightarrow{\mathbf{G}}^{k}$, the balancing torque; $\epsilon_{i j k}$ is the permutation symbol; $\lambda_{1}$ and $\lambda_{2}$ are the associated penalty parameters whose magnitude controls the speed of convergence. The larger the penalty parameter, the faster the convergence rate. Because of the explicit schemes are implemented, the magnitude is subject to the stability condition. In reality, it only takes a few iterations between two consecutive time steps. Upon convergence, the nominal velocity of the $k^{\text {th }}$ cylinder $\mathbf{V}^{k}(t, \tau)$ becomes zero, and the unknown balancing force becomes independent of $\tau$, and the value for $\mathbf{F}_{B}^{k}(t, \tau)$ is obtained. Similarly, the balancing torque $\overrightarrow{\mathbf{G}}^{k}$ with component $T_{i j}$ acting in the plane $i j$, is computed from the angular velocity $\Omega_{k}(t, \tau)$ of this cylinder. Once these forces and torque from each cylinder to the fluid are updated, they are used in the Navier-Stokes equations to 
solve for the flow field at the next time step. This penalty algorithm applies to both resistance and mobility problems.

\subsection{Lagrangian Description of Cylinders}

The nominal velocity of each cylinder $\mathbf{V}^{k}(t)$ is computed from nonlinear filtering, i.e., a volume integral of the fluid velocity with the kernel function:

$$
\mathbf{V}^{k}(t)=\iiint_{V o l} \mathbf{u}(\mathbf{x}, t) \Phi\left[\mathbf{x}-\mathbf{Y}^{k}(t), \sigma, t\right] d V o l .
$$

Similarly, the nominal angular velocity $\boldsymbol{\Omega}^{k}(t)$ of the cylinder is computed with a volume integral of the fluid vorticity $\nabla \times \mathbf{u}(\mathbf{x}, \mathrm{t})$ with the kernel:

$$
\boldsymbol{\Omega}^{k}(t)=\frac{1}{2} \iiint_{V o l} \nabla \times \mathbf{u}(\mathbf{x}, t) \Phi\left[\mathbf{x}-\mathbf{Y}^{k}(t), \sigma, t\right] d V o l .
$$

Finally, the nominal translational position and rotational angle of the cylinder could be computed explicitly from the Lagrangian description:

$$
d \mathbf{Y}^{k}(t)=\mathbf{V}^{k}(t) d t, \quad d \mathbf{A}^{k}(t)=\mathbf{\Omega}^{k}(t) d t .
$$

The problems here are resistance problems [42] in multiphase flows. Therefore, the translational and rotational velocities are zero as cylinders are fixed. However, during the computations in the penalty algorithm, they were penalized to be nonzero until convergence.

\subsection{Discretization Method and Integration Schemes}

The modal spectral element method with $h p$ resolution refinement, developed by Warburton, Sherwin, and Karniadakis [43,44] was implemented in space for all VCM and DNS simulations. This method inherits the exponential convergence of spectral methods and discretizes complex geometries with regular finite elements. A high order splitting algorithm [45] was used in time to obtain numerical solutions to the three-dimensional incompressible Navier-Stokes equations. Gauss-Lobatto-Legendre quadratures were extensively used to numerically evaluate volume integrals in VCM computations. Adam-Bashforth schemes of third order accuracy were used to integrate Lagrangian equations for velocities of cylinders. 

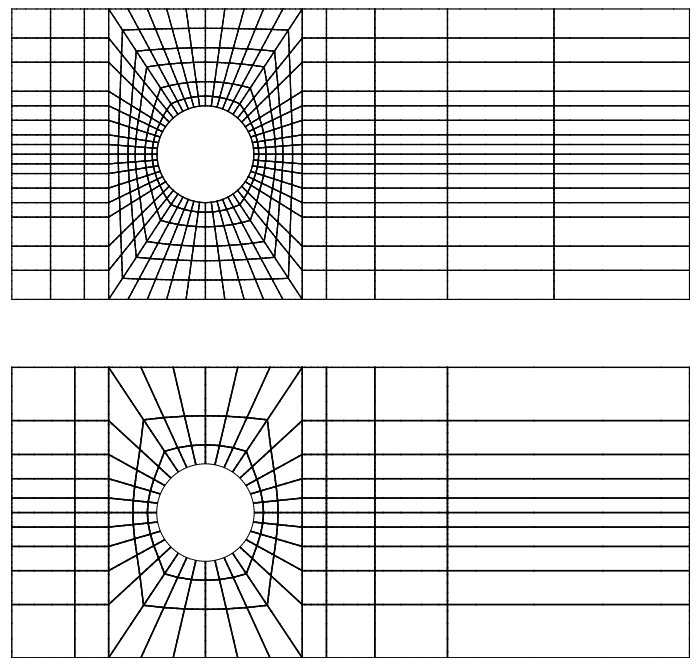

(a)
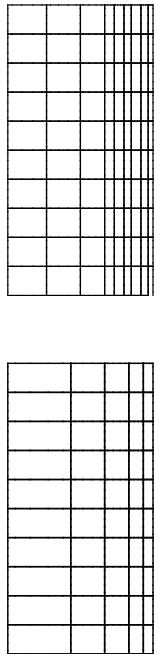

(b)

Fig. 1. Configuration of spatial elements used in DNS mesh in (a) the plane $x=0$ and (b) the plane $\mathrm{y}=0$ : 4,400 elements (upper plots) and 1,560 elements (lower plots). The fluid enters horizontally along $z$ from the left plane and exits from the right plane.

\section{Validation and Discussion}

\subsection{Flow Past One Row of Periodic Cylinders}

We consider one row of long cylinders fixed still vertically and evenly in a uniform flow in an unbounded domain. The radii of these cylinders are $a=1$. The spacing between them in the lateral $(y)$ direction is $6 a$, which is the distance between center lines of two adjacent cylinders. Due to the geometric periodicity, we focus on the rectangular volume of dimensionless size $-3 \leq x / a \leq 3,-3 \leq y / a \leq 3$, and $-4 \leq z / a \leq 10$ containing one cylinder with its axis of symmetry parallel to $x$ at $y / a=0$ and $z / a=0$. We consider long cylinders and ignore the end effect. Therefore, periodic boundary conditions were applied at the ends in $x$ along the length of the cylinder and in $y$ laterally. To drive the flow in $z$, a constant uniform flow of dimensionless velocity $w=10$ was specified as a Dirichlet boundary condition in the plane $z=-4$ on the left (see Fig. 1). A Neumann boundary condition $\partial w / \partial z=0$ was imposed at the exit in the plane $z=10$ on the right. Both the density $\rho$ and dynamic viscosity $\mu$ of the fluid were scaled to 1 . The Reynolds number based on the approach velocity and cylinder diameter is 20 . The cylinder is fixed stationary, no translation and rotation, by external force and torque, which are unknown prior to DNS and VCM runs. This is a resistance problem in fluid mechanics point of view.
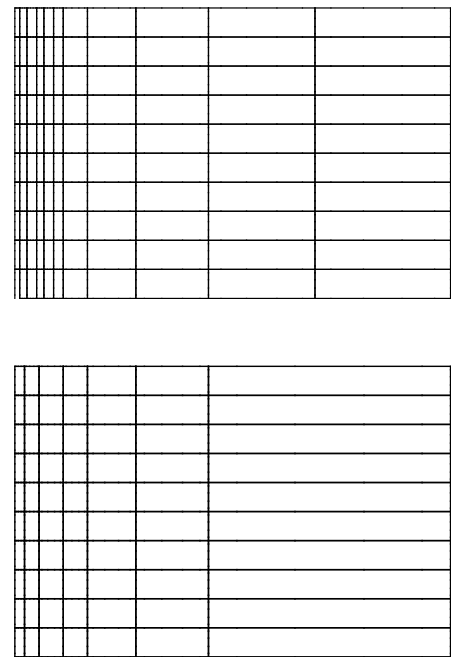

Direct numerical simulation (DNS) runs were conducted to provide reliable solution for verification purpose for simulations with VCM (VCM runs for short). In DNS, the Navier Stokes equations were solved with nonslip boundary conditions imposed on the cylindrical surface. Therefore, accurate drag force on the cylinder could be obtained. 


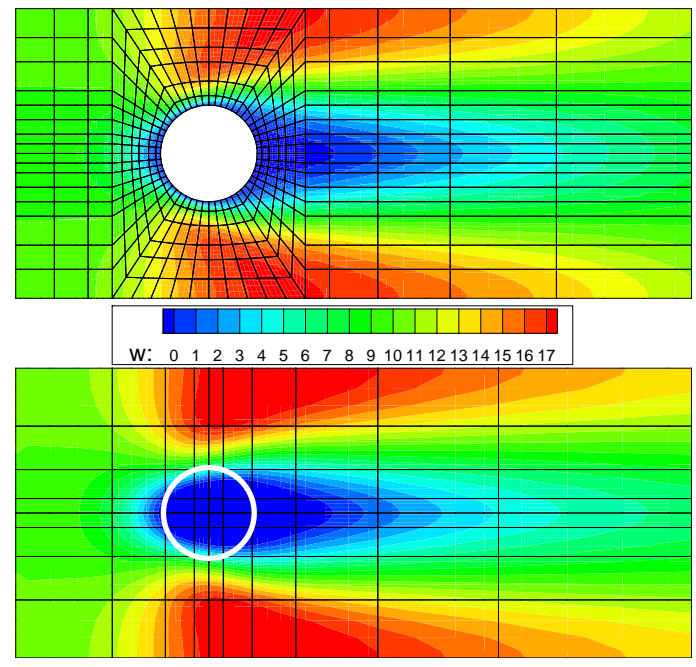

(a)
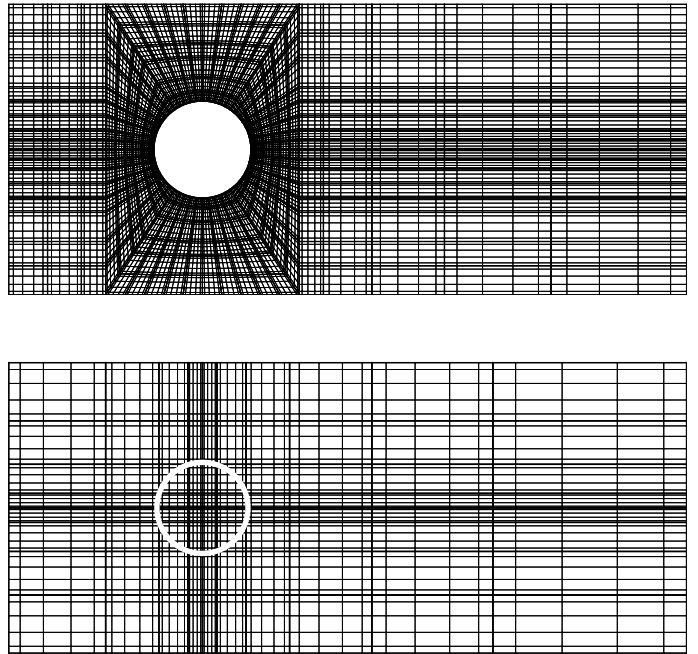

(b)

Fig. 2. Comparisons of (a) mainstream fluid velocity and the background spatial elements; (b) Elements and interior quadrature points. Both (a) and (b) are in the z-y plane through the middle of the cylinder: DNS on 4,400 elements (upper plots); VCM on 800 elements (lower plots). The circle in the lower plot denotes the location of the cylinder.

VCM runs take much less time than DNS and are especially suitable for flows involving numerous cylinders. In a VCM run for flow over a periodic cylinder, the nonslip boundary conditions are not imposed, instead, a force field is imposed in the momentum equation of the fluid. This force field has its magnitude iteratively computed with the penalty method described before and is distributed with a kernel function in the space where the cylinder has an effective influence. The obvious advantage is that the computational mesh does not need to be body-fitting with tiny elements surrounding the cylinder; elements fill the entire rectangular volume, see the lower plot in Figure 2 (b). The number of elements in VCM runs are much less than DNS; Therefore, VCM runs are significantly faster than DNS. This is ideal for applications with numerous moving cylinders. Results from VCM runs were obtained using 480 and 800 hexahedral elements, respectively, at $3^{\text {rd }}$ to $5^{\text {th }}$ order polynomials basis expansions. The lower plots in Figure 2 present the mainstream fluid velocity from VCM on 800 elements. At a much less cost in space and time, VCM results are quite similar to 
Table 1

Comparison of the drag force (DNS) and the balancing force (VCM) at varied spatial resolutions

\begin{tabular}{|c||c|c|c|c|}
\hline DNS & 1,560 elem., 3rd & 1,560 elem., 4th & 1,560 elem., 5th & 4,400 elem., 5th \\
\hline Drag & $2686.759(0.8 \%)$ & $2703.120(0.2 \%)$ & $2708.389(0.03 \%)$ & $\mathbf{2 7 0 9 . 0 8 7}$ \\
\hline \hline VCM & 480 elem., 4th & 480 elem., 5th & 800 elem., 4th & 800 elem., 5th \\
\hline Drag & $-2729.753(0.8 \%)$ & $-2723.804(0.5 \%)$ & $-2728.358(0.7 \%)$ & $-2724.639(0.6 \%)$ \\
\hline
\end{tabular}

Table 2

Comparison of wall-clock time (Time) and approximate degrees of freedom (DOF) for DNS and VCM runs in Table 1

\begin{tabular}{|c||c|c|c|c|}
\hline DNS & 1,560 elem., 3rd & 1,560 elem., 4th & 1,560 elem., 5 th & 4,400 elem., 5 th \\
\hline DOF & $100 \mathrm{k}$ & $195 \mathrm{~K}$ & $340 \mathrm{k}$ & $950 \mathrm{k}$ \\
\hline Time & $0: 25: 57$ & $0: 38: 50$ & $2: 06: 24$ & $17: 28: 18$ \\
\hline \hline VCM & 480 elem., $4 \mathrm{th}$ & 480 elem., 5 th & 800 elem., 4 th & 800 elem., 5 th \\
\hline DOF & $60 \mathrm{k}$ & $104 \mathrm{~K}$ & $100 \mathrm{k}$ & $172 \mathrm{k}$ \\
\hline Time & $0: 22: 47$ & $1: 02: 25$ & $0: 45: 51$ & $2: 36: 21$ \\
\hline
\end{tabular}

DNS.

Table 1 tabulates the drag force from DNS runs and the external force on the cylinder to balance the drag force in $z$ from VCM runs. Values are all in dimensionless units. An extra DNS run with 4,400 elements at $9^{\text {th }}$ order expansion with a parallel algorithm was also performed on 48 Linux cores using MPI to provide even higher resolution. The drag force came out to be 2,709.348. This run was not included here because it is a parallel algorithm. In Table 1, the most reliable value 2,709.087 for the drag force was from the DNS run on 4,400 elements at $5^{\text {th }}$ order expansion. The relative errors to this value for other runs were listed in parentheses next to the drag value. It is justified that using 1,560 elements with $3^{\text {rd }}$ or higher order expansion could generate mesh-independent drag force. Since those computed external forces from VCM are to balance drag forces in DNS, by Newton's third law of motion, values of balancing forces in VCM runs are negative (same as below), as shown in Figure 3 (a). The computational time in DNS is about $5 \sim 10$ of that of VCM runs.

Table 2 presents the approximate degrees of freedom (DOF) and the wall-clock time used in eight cases listed in Table 1 . All runs in Table 1 were performed on a single Linux (Fedora 15) machine with Intel i-7 quad-core processors and 32GB RAM. Due to the iterative algorithm implemented in VCM for the balancing force, the pseudotime loop between two consecutive time steps took a little more time than without the iterative algorithm. Comparing DNS and VCM runs at the highest resolution, i.e., 4,400 elements in DNS and 1,560 elements in VCM both at $5^{\text {th }}$ order expansion, the DNS required about 5.5 times higher resolution than the VCM. However, the 


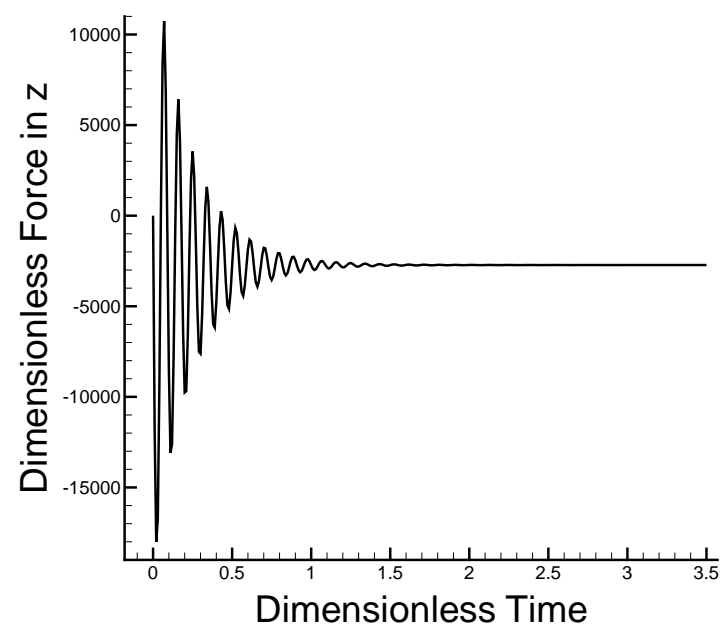

(a)

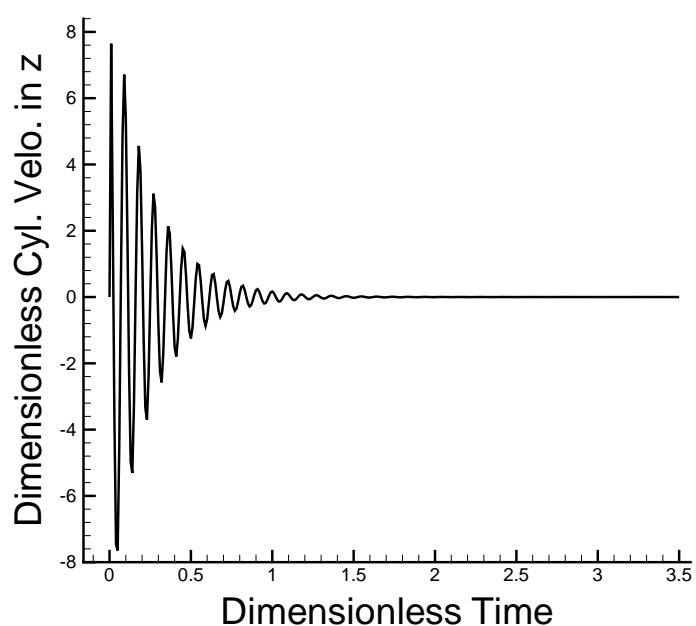

(b)

Fig. 3. Time evolution of (a) the balancing drag; (b) the computed cylinder velocity

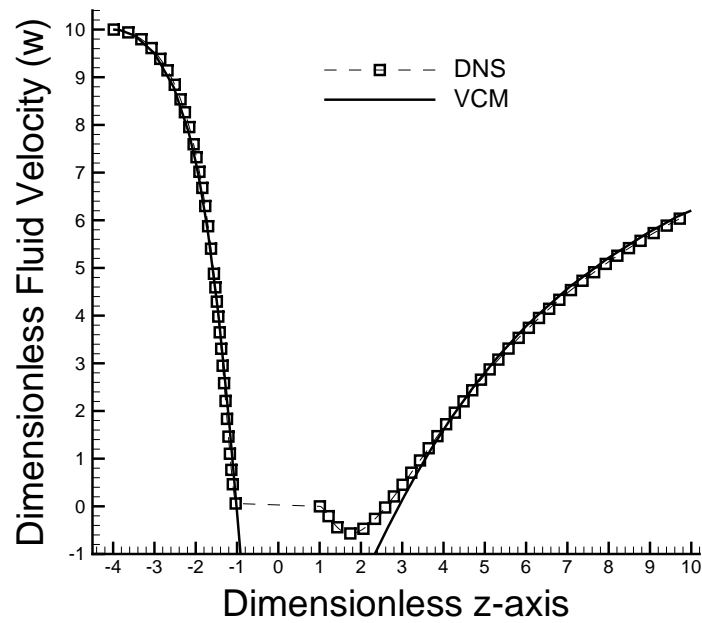

(a)

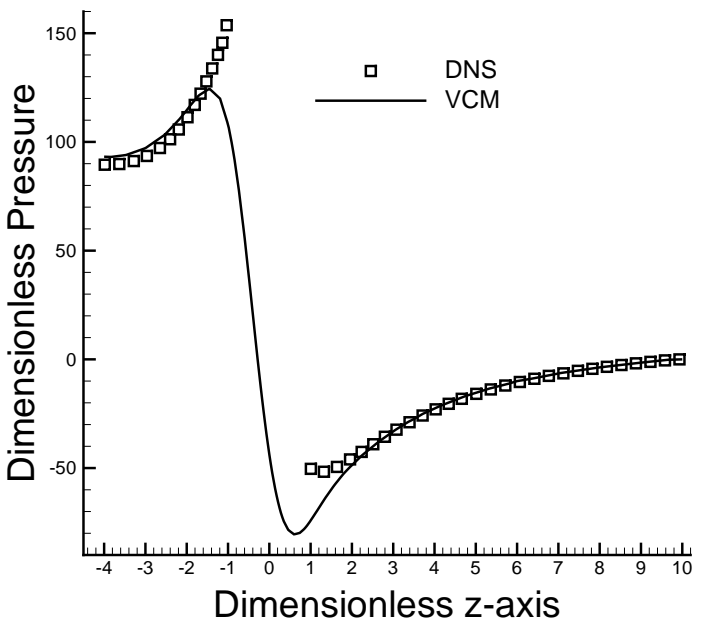

(b)

Fig. 4. Comparisons of mainstream fluid $w$-velocity (a) and pressure (b) versus the main flow $z$ direction along the line $y=0$ and $x=0$

To demonstrate the success of the penalty method for computing the balancing force der velocity are shown in Figure 3. Although both plots show similar oscillation 
towards final convergence, actually they are out of phase in time. The convergence rate was controlled by the penalty parameter whose magnitude was restricted by the stability condition since explicit methods of time integration were used to compute the balancing force in VCM runs.

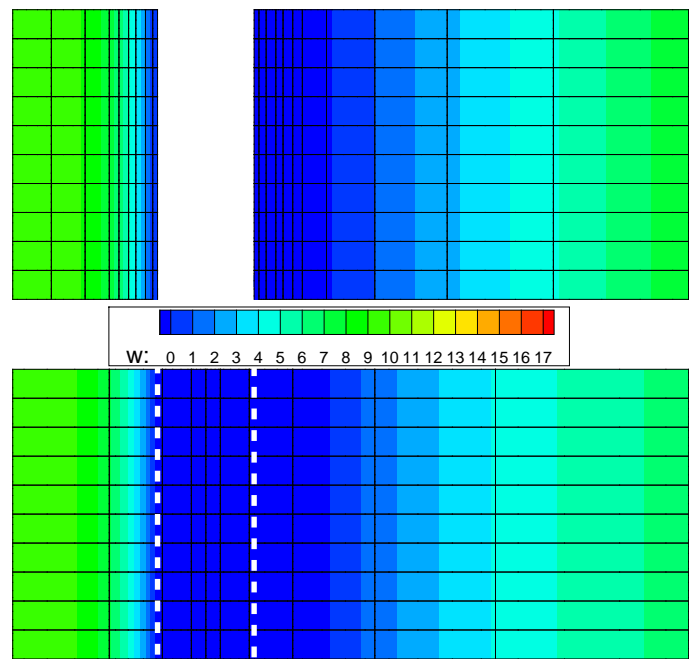

(a)
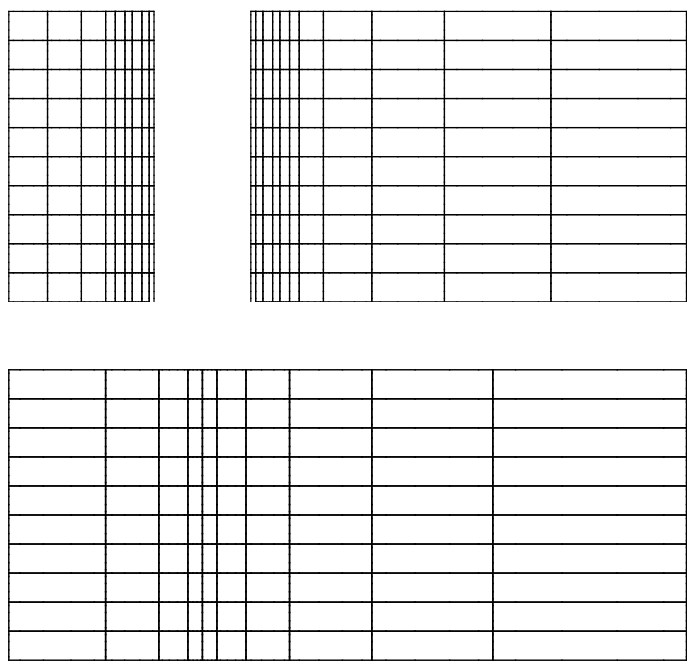

(b)

Fig. 5. Comparisons of (a) the mainstream fluid velocity in the plane $y=0$; and (b) spatial elements in DNS (upper) and VCM (lower)

Figure 4 compares the mainstream fluid velocity (a) and pressure (b) along the mainstream $(z)$ direction, between DNS and VCM runs. The small discrepancy near the surface of the cylinder, $-1 \leq z \leq 1$, was due to the fact that VCM runs do not use body-fitted mesh near the cylinder and nonslip boundary conditions were not imposed at all on the cylindrical surface. However, the integral-averaged drag force in VCM is accurate, though at the price of resolving the fluid volume inside the cylinder.

Figure 5 (a) compares the mainstream fluid velocity $w$ in the z-x plane. The distribution of elements for both DNS (upper) and VCM (lower) are shown in Figure 5 (b). In this paper, only the fluid motion in the z-y plane are considered and the motion in $x$ is reserved for a subsequent paper which investigates tilted and bendable cylinders which will receive a torque about $y$ axis near its end point along $x$. To eliminate the influence of the spatial $(x)$ resolution on numerical solutions, the number and size of elements were kept the same for all related DNS and VCM runs in this work.

Next we turn onto the interaction of two cylinders in the tandem configuration.

\subsection{Flow Past Two Rows of Aligned Periodic Cylinders}

Two rows of stationary cylinders are regularly aligned in a uniform and unbounded flow. The radii of cylinders are $a=0.5$ dimensionless unit (same hereafter). The dis- 
tance between centers of two neighboring cylinders is $6 a$ in both the mainstream $z$ and the lateral $y$ directions. Due to the geometric periodicity, we focus on the rectangular volume of size $0 \leq x \leq 2,-1.5 \leq y \leq 1.5$, and $0 \leq z \leq 6$ containing only two cylinders in the tandem configuration. The center-lines of cylinders are parallel to $x$ and through two points $(0,0,1.5)$ and $(0,0,4.5)$, respectively. The end effect are ignored and periodic boundary conditions were imposed at planes $x=0, x=2$, and laterally at $y= \pm 1.5$ between neighboring cylinders. A constant uniform flow of velocity $w=10$ along the positive $z$ direction was specified as a Dirichlet boundary condition in the plane $z=0$ on the left, as shown in Fig. 6. A Neumann boundary condition $\partial w / \partial z=0$
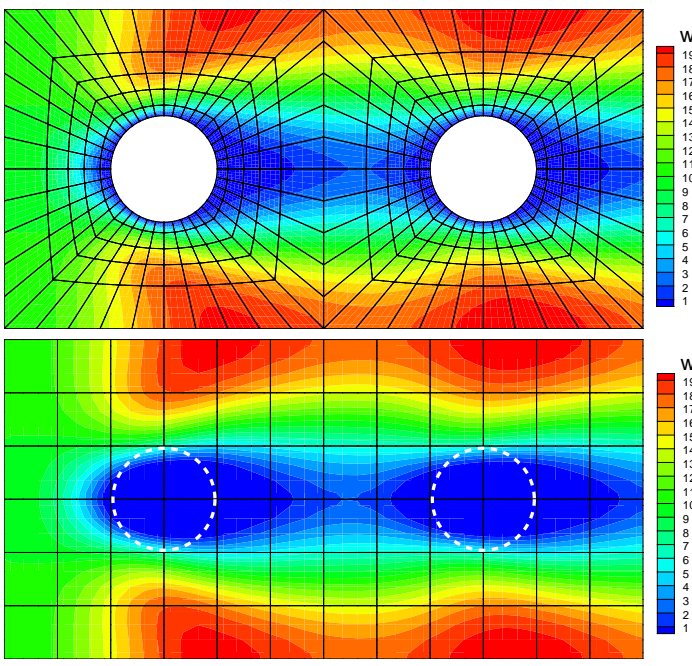

(a)
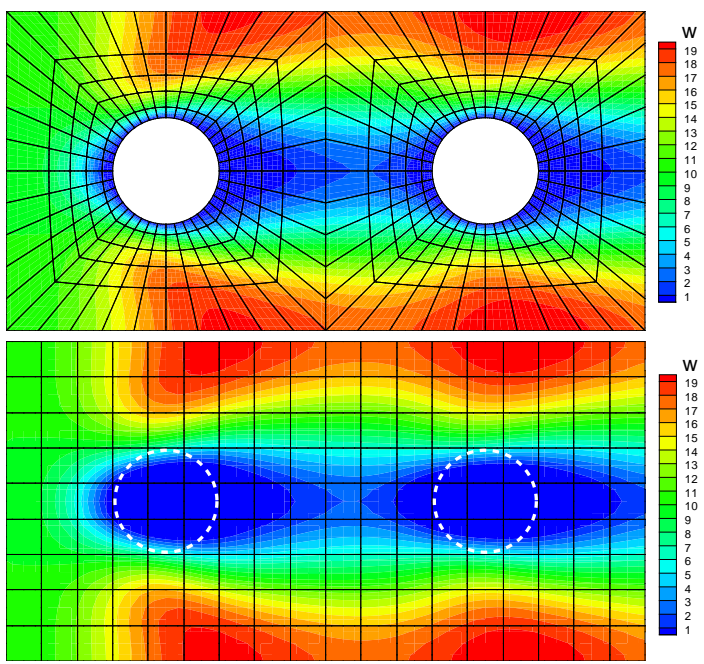

(b)

Fig. 6. Comparisons of the mainstream fluid velocity in the plane $x=1$ between the DNS run (upper) on 2,000 elements at $6^{\text {th }}$ order expansion and VCM runs (lower) on: (a) 360 elements at $5^{\text {th }}$ order expansion; (b) 810 elements at $4^{\text {th }}$ order expansion, respectively

was imposed at the exit plane $z=6$ on the right. Both the density $\rho$ and dynamic viscosity $\mu$ of the fluid were scaled to 1 . The Reynolds number based on the approach velocity for the leading cylinder and its diameter is 10. External forces that maintain cylinders stationary without any translation or rotation were unknown prior to both DNS and VCM runs. This is again categorized as a resistance problem.

To validate VCM runs, a set of DNS runs were completed in which small cylindrical surface-conforming hexahedral elements were used to enhance the resolution near two cylinders and nonslip boundary conditions were imposed on cylindrical surfaces. Larger elements were used in the far field. After some optimization, the number of elements were reduced to 2,000, as shown in the upper plots in Figure 6. The NavierStokes equations were numerically solved at the $3^{\text {rd }}$ to $6^{\text {th }}$ order polynomial expansion in all directions in all elements. Four sets of DNS runs at varied resolutions were obtained and accurate drag forces on the leading cylinder, i.e., cylinder 1 are listed in Table 3. Drag forces on cylinder 2 are listed in Table 4. The leading cylinder has experienced a larger drag force than the trailing one. The differences in drag between different runs are less than $2 \%$ relative to the data of highest resolution highlighted 
Table 3

Comparison of the drag force (DNS) with the balancing force (VCM) at varied spatial resolutions for cylinder 1 (leading) at $y=0, z=1.5$

\begin{tabular}{|c||c|c|c|c|}
\hline DNS & 2,000 elem., 3rd & 2,000 elem., 4th & 2,000 elem., 5th & 2,000 elem., 6th \\
\hline Drag & $711.933(0.7 \%)$ & $707.611(0.09 \%)$ & $707.069(0.01 \%)$ & $\mathbf{7 0 6 . 9 8 9}$ \\
\hline \hline VCM & 360 elem., 4th & 360 elem., 5th & 360 elem., 6th & 810 elem., 4 th \\
\hline Drag & $-709.984(0.4 \%)$ & $-710.299(0.5 \%)$ & $-710.350(0.5 \%)$ & $-710.207(0.5 \%)$ \\
\hline
\end{tabular}

Table 4

Comparison of the drag force (DNS) and the balancing force (VCM) at varied spatial resolutions for cylinder 2 (trailing) at $y=0, z=4.5$

\begin{tabular}{|c||c|c|c|c|}
\hline DNS & 2,000 elem., 3rd & 2,000 elem., 4th & 2,000 elem., 5th & 2,000 elem., 6th \\
\hline Drag & $504.912(1.1 \%)$ & $500.192(0.12 \%)$ & $499.694(0.02 \%)$ & $\mathbf{4 9 9 . 5 7 2}$ \\
\hline \hline VCM & 360 elem., 4th & 360 elem., 5th & 360 elem., 6th & 810 elem., 4th \\
\hline Drag & $-496.647(0.6 \%)$ & $-496.948(0.5 \%)$ & $-496.825(0.5 \%)$ & $-496.769(0.6 \%)$ \\
\hline
\end{tabular}

in these tables. This indicated that the DNS mesh with 2,000 elements is reliable and has sufficient resolution for resolving boundary layers.

VCM runs were performed with better efficiency (less wall clock time than DNS) at acceptable accuracy. Similar to the previous section, nonslip boundary conditions were not imposed on cylindrical surfaces, instead, an iteratively computed force field was imposed in the momentum equation of the fluid and distributed via a kernel function. The advantage of avoiding tiny curvature-fitting elements surrounding cylinders is shown here with significantly less number of hexahedral elements in VCM runs than in DNS runs. This is the major reason that VCM runs are much faster than corresponding DNS runs. Two different VCM meshes with 360 and 810 elements were used for solutions at $4^{\text {th }}$ through $6^{\text {th }}$ order polynomial expansions. Lower plots in Figure 6 show the fluid velocity contour lines in the plane $x=1$ obtained from (a) 360 elements and (b) 810 elements. Values of drag force for the leading and trailing cylinder are separately listed in Table 3 and Table 4 . The percentages within parentheses are errors relative to the most reliable DNS data (in bald face). Using only a fraction of the number of elements in DNS, VCM runs acquired drag force of accuracy comparable to DNS with errors less than 1\%. This serves as another demonstration of the efficiency of VCM.

Figure 6 presents the comparison of the mainstream fluid velocity between DNS and VCM runs using different number of elements and order of expansions in the z-y plane at $\mathrm{x}=1$. Compared to 400 elements used in DNS in the plane $x=1$, the VCM run in Figure 6 (a) used only 72 elements and the other VCM run in Figure 6 (b) used 162 elements. The efficiency of VCM was due to avoiding tiny surface-fitting elements and using larger and coarse elements. However, the non-uniformly distributed quadrature points inside each element, not shown in the figure, are capable of resolving sharp 
Table 5

Comparison of wall-clock time (Time) and approximate degrees of freedom (DOF) for DNS and VCM runs in Table 3 and Table 4

\begin{tabular}{|c||c|c|c|c|}
\hline DNS & 2,000 elem., 3rd & 2,000 elem., 4th & 2,000 elem., 5th & 2,000 elem., 6th \\
\hline DOF & $128 \mathrm{k}$ & $250 \mathrm{~K}$ & $432 \mathrm{k}$ & $686 \mathrm{k}$ \\
\hline Time & $0: 09: 57$ & $0: 38: 50$ & $2: 06: 24$ & $8: 18: 24$ \\
\hline \hline VCM & 360 elem., 4th & 360 elem., 5 th & 360 elem., 6 th & 810 elem., $4 \mathrm{th}$ \\
\hline DOF & $45 \mathrm{k}$ & $78 \mathrm{~K}$ & $124 \mathrm{k}$ & $101 \mathrm{k}$ \\
\hline Time & $0: 06: 41$ & $0: 31: 15$ & $2: 21: 02$ & $0: 16: 02$ \\
\hline
\end{tabular}
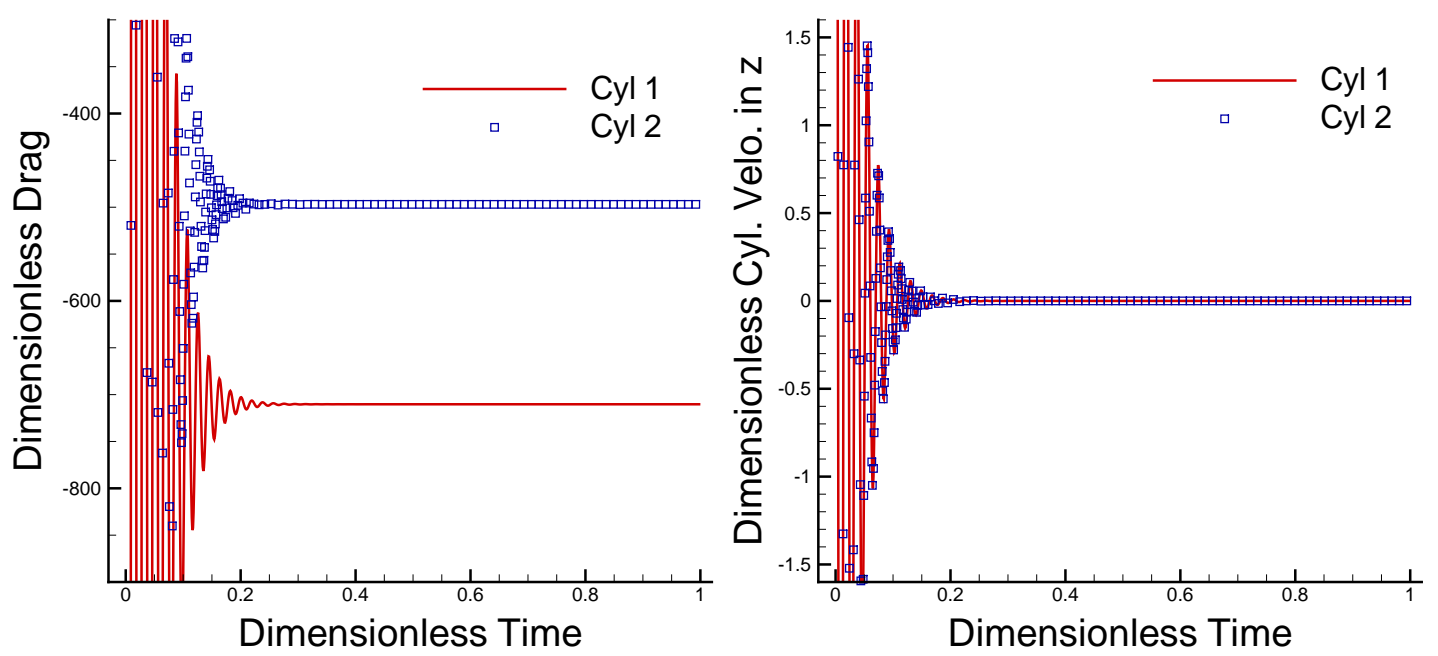

Fig. 7. Evolution of the balancing drag forces (left) and the computed cylinder velocity values (right) for cylinder 1 at $\mathrm{y}=0, \mathrm{z}=1.5$ and cylinder 2 at $\mathrm{y}=0, \mathrm{z}=4.5$

gradients in the field. The dashed circles in plots denote the location of cylinders. These plots indicate that using much less elements, the VCM model could still capture the fundamental characteristics of the flow field. Due to the interference from the trailing cylinder, the wake of the leading cylinder is slightly short than cylinder 2 . The drag force on cylinder 1 is larger than cylinder 2. This is consistent with the extensive experimental study by Zdravkovich [16].

The VCM runs outperform the DNS runs in terms of the computational cost. Table 5 presents the approximate degrees of freedom (DOF) and the wall-clock time in all runs listed in Tables 3 and 4 . These runs were accomplished on a single Linux (Fedora 15) machine with Intel i-7 quad-core processors and 32GB RAM. Because VCM runs use an iterative algorithm between time steps to compute the balancing force for the drag, a little more wall clock time was used than otherwi without iterations. The differences in drag force between DNS runs are less than $1 \%$. This indicates that DNS runs were fully converged. DNS runs used 3 6 times more elements and 3 6 times more DOF than VCM runs. Therefore, the wall clock time for a VCM run is only a fraction of that for a DNS run, as seen in the table. 


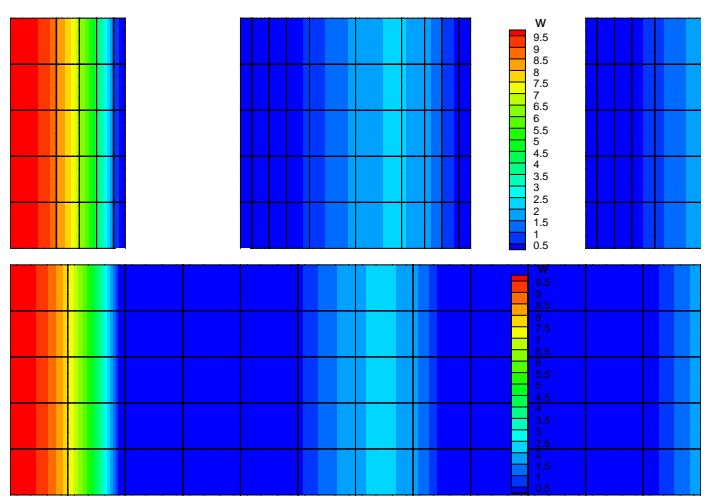

(a)
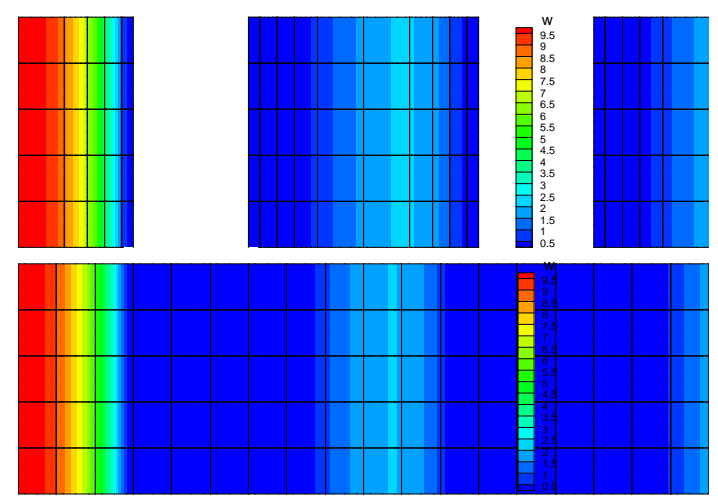

(b)

Fig. 8. Comparison of the mainstream fluid velocity in the plane $y=0$ between the DNS run (upper) on 2,000 elements at $6^{\text {th }}$ order expansion and VCM runs (lower) on: (a) 360 elements at $5^{\text {th }}$ order expansion; (b) 810 elements at $4^{\text {th }}$ order expansion, respectively
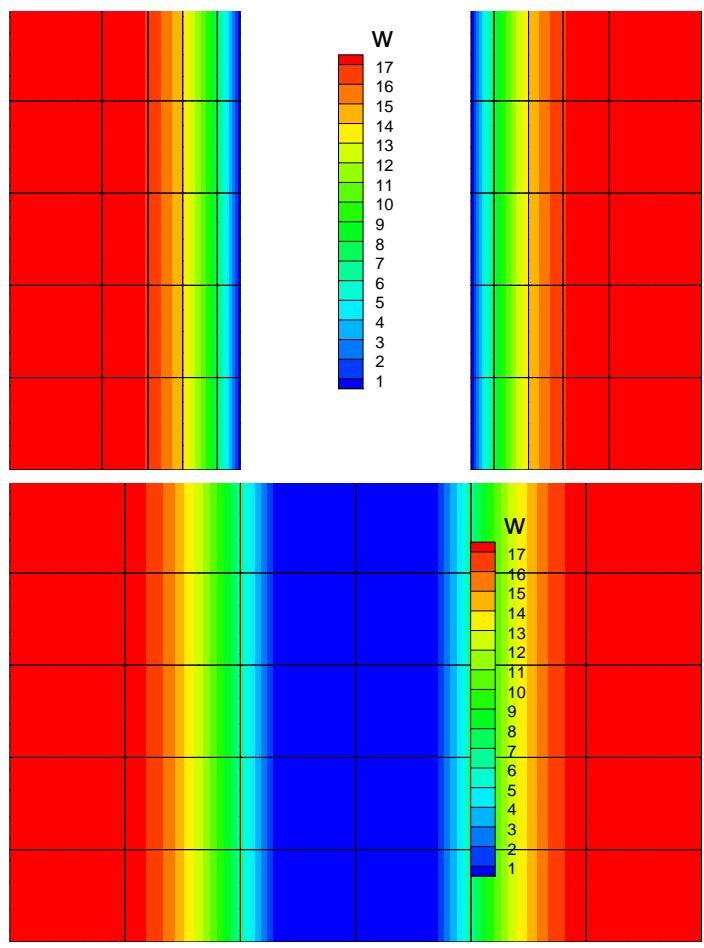

(a)
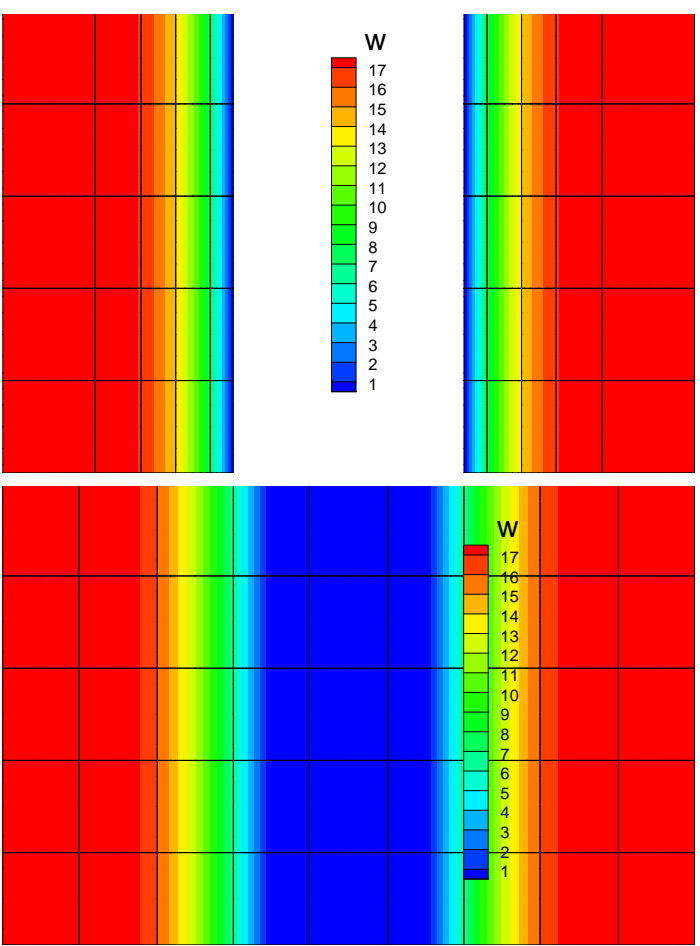

(b)

Fig. 9. Comparison of the mainstream fluid velocity in the plane $z=1.5$ between the DNS run (upper) on 2,000 elements at $6^{\text {th }}$ order expansion and VCM runs (lower) on: (a) 360 elements at $5^{\text {th }}$ order expansion; (b) 810 elements at $4^{\text {th }}$ order expansion, respectively

The left plot in Figure 7 shows the convergence history of the drag-balancing force in VCM run on 360 elements at $5^{\text {th }}$ order polynomial expansion. As previously mentioned, the balancing forces are negative. From time being 0 to 0.2 dimensionless unit, the penalized balancing force seeks to satisfy the velocity constraint and oscillates around the final value with a fast damping. Convergence was quickly reached 
with a large but stability-preserving penalty parameter. The right plot in Figure 7 demonstrates the constrained velocity computed from integral evaluations. The converged values become zero as cylinders are stationary.

Figure 8 shows the comparison of the mainstream fluid velocity between DNS and VCM runs using different numbers of elements and orders of expansions in the $\mathrm{z}-\mathrm{x}$ plane at $y=0$. Due to the fact that the plane $y=0$ is through the axes of symmetry of two vertical cylinders, there are two empty rectangles in the upper plots for the DNS run. In contrast, in the same area in the lower plots for VCM runs, the fluid is almost in stagnation, although it could detour around these two virtual cylinders. Overall, the patterns of fluid velocity distribution are similar, even if nonslip boundary conditions were not imposed in VCM runs at all.

Figure 9 compares the mainstream fluid velocity between DNS and VCM runs at different resolutions in the $y$-x plane at $z=1.5$ through the axis of symmetry of cylinder 1. The empty rectangle in the upper plots is the cross sectional area of cylinder 1 . In the lower plots for VCM runs, inside the volume nominally occupied by cylinder 1, the fluid is actually flowing backwards, seemingly in stagnation in plots. If we just focus on the flow outside the cylinder, then there is not much difference between DNS and VCM runs. These plots indicate similar detouring patterns and velocity magnitude of the fluid. With the advantage of avoiding resolving boundary layer of cylinders, VCM runs could simulate many cylinders at much lower computational cost than performing DNS with a parallel algorithm.

In summary, VCM runs compute the drag force by penalizing the cylinder velocity which quickly converges to zero, distribute the force field of the computed force which balances the drag, and only solve the Navier-Stokes equation on a fixed mesh of relatively low resolution than that of the DNS. Although in VCM runs the boundary layer around the cylinder is not resolved, its interior volume is completely resolved instead as a part of the computational domain. Due to the relief of resolving boundary layer near curved surface of an object in the fluid, VCM runs are significantly faster than the corresponding DNS at the same level of accuracy.

\section{Applications}

After validating VCM runs with corresponding DNS runs and observing the gain in terms of the computational cost when VCM algorithm was used, we could use VCM for runs that DNS and other methods could become significantly slow. 


\subsection{Flow Past Eight Randomly Arranged Cylinders}

With eight cylinders of radii $a=0.5$ dimensionless unit (same hereafter) randomly arranged in a three dimensional flow between two parallel walls, the flow field is asymmetrical in both the mainstream and lateral directions due to the disturbance from these randomly located cylinders. Two walls are located at $y= \pm 3$, respectively, and are 6 units apart in $y$. Each wall measures from 0 to 2 in $x$ and from 0 to 12 in $z$, with nonslip boundary conditions imposed. At the fluid entrance, i.e. the plane $z=0$, a fully developed Poiseuille flow was specified so that the peak velocity along the center plane $y=0$ is $w=10$ before disturbed by cylinders. The peak Reynolds number based on cylinder diameter is 15 . At the exit plane $z=12$, a Neumann boundary condition $\partial w / \partial z=0$ was imposed. Along the length of cylinders in $x$, periodic boundary conditions were specified at $x=0$ and 2, respectively, to eliminate the end effect of cylinders. Both the density $\rho$ and dynamic viscosity $\mu$ of the fluid were scaled to 1 . This is another resistance problem as all cylinders are free from any motion.

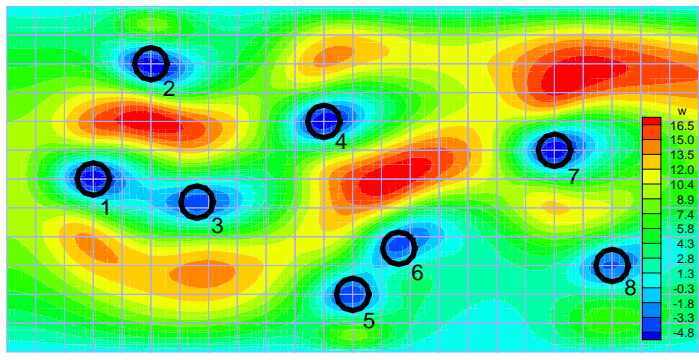

(a)

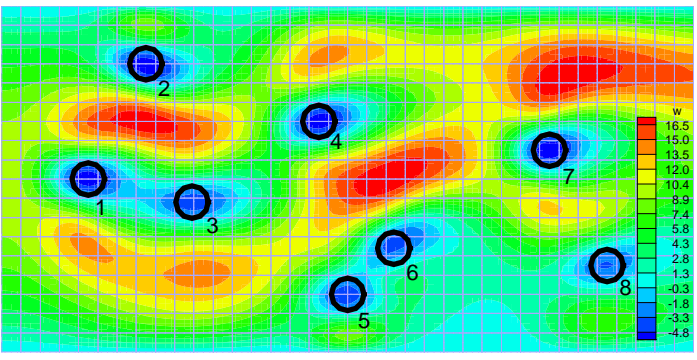

(b)

Fig. 10. Comparison of the mainstream (z) fluid velocity and spatial elements in VCM runs on: (a) 576 elements at $6^{\text {th }}$ order expansion and, (b) 1,296 elements at $4^{\text {th }}$ order expansion. Locations of cylinders are digitally indicated.

Performing DNS requires hundreds of thousands of tiny curved surface-conforming elements near cylinders to resolve boundary layers for drag forces. Although we have the capability of performing such kind of large scale DNS with a parallel algorithm, it will be very expensive to complete sets of mesh-independent DNS runs. For this reason, only VCM runs at varied resolutions were completed here. The computational domain was discretized with two separate elemental configurations. The first mesh has 2 elements in $x, 12$ in $y$, and 24 in $z$, for a total of 576 hexahedral elements. The second mesh has 2 in $x, 18$ in $y$, and 36 in $z$, for a total of 1,296 hexahedral elements. Within each element, $3^{\text {rd }}$ through $6^{\text {th }}$ order polynomial expansions in each direction were used. Figure 10 (a) shows the mainstream fluid velocity on 576 elements at $6^{\text {th }}$ order expansion. Figure 10 (b) is similar but on 1,296 elements at $4^{\text {th }}$ order expansion. The actual elements were shown in the background for both plots. The locations of cylinders were marked with black circles and labeled from 1 to 8. Figure 10 (a) has a slightly higher resolution with approximately 198,000 degrees of freedom, compared with Figure 10 (b) with 162,000 degrees of freedom, due to more interior quadrature 


\section{Table 6}

Comparison of drag and lift forces on all cylinders from four different computations on two separate sets of mesh: 576 elements and 1,296 elements

\begin{tabular}{|c||c|c|c|c|}
\hline Drag/Lift & 576 elem., 4th & 576 elem., 5th & 576 elem., 6th & 1,296 elem., 4th \\
\hline \hline Cyl. 1 & $623.93 /-93.23$ & $624.14 /-93.14$ & $624.78 /-90.72$ & $624.07 /-93.13$ \\
\hline Cyl. 2 & $746.08 /-242.65$ & $746.25 /-243.03$ & $745.68 /-243.59$ & $746.12 /-242.95$ \\
\hline Cyl. 3 & $383.54 / 38.90$ & $383.50 / 38.93$ & $383.68 / 39.03$ & $383.46 / 38.93$ \\
\hline Cyl. 4 & $624.00 / 174.75$ & $624.64 / 174.91$ & $624.77 / 175.14$ & $624.38 / 174.83$ \\
\hline Cyl. 5 & $660.22 /-52.01$ & $661.19 /-52.32$ & $660.26 /-53.55$ & $660.88 /-52.22$ \\
\hline Cyl. 6 & $453.35 / 191.35$ & $453.62 / 191.33$ & $454.54 / 193.37$ & $453.37 / 191.27$ \\
\hline Cyl. 7 & $645.83 / 34.17$ & $646.97 / 34.15$ & $651.11 / 31.79$ & $646.53 / 34.17$ \\
\hline Cyl. 8 & $358.05 / 38.23$ & $358.68 / 38.29$ & $361.98 / 37.28$ & $358.45 / 38.27$ \\
\hline
\end{tabular}

Table 7

Comparison of torque values on all cylinders from four different computations on two separate sets of mesh: 576 elements and 1,296 elements

\begin{tabular}{|c||c|c|c|c|}
\hline Torque & 576 elem., 4th & 576 elem., 5th & 576 elem., 6th & 1,296 elem., 4th \\
\hline \hline Cyl. 1 & 5.85 & 5.81 & 5.68 & 5.80 \\
\hline Cyl. 2 & -44.65 & -44.53 & -44.32 & -44.53 \\
\hline Cyl. 3 & -9.11 & -9.13 & -9.14 & -9.13 \\
\hline Cyl. 4 & 0.99 & 0.96 & 0.96 & 0.97 \\
\hline Cyl. 5 & -17.66 & -17.59 & -17.07 & -17.57 \\
\hline Cyl. 6 & 80.11 & 80.19 & 80.05 & 80.18 \\
\hline Cyl. 7 & -3.96 & -4.01 & -3.79 & -4.00 \\
\hline Cyl. 8 & -2.62 & -2.64 & -2.90 & -2.64 \\
\hline
\end{tabular}

Table 8

Wall clock time (Time) and approximate degrees of freedom (DOF) for VCM runs

\begin{tabular}{|c||c|c|c|c|}
\hline VCM & 576 elem., 4th & 576 elem., 5 th & 576 elem., 6th & 1,296 elem., 4th \\
\hline \hline DOF & $72 \mathrm{k}$ & $125 \mathrm{~K}$ & $198 \mathrm{k}$ & $162 \mathrm{k}$ \\
\hline Time & $0: 08: 17$ & $0: 47: 05$ & $2: 29: 07$ & $1: 21: 04$ \\
\hline
\end{tabular}

Table 6 gives the computed drag and lift force, in dimensionless units, on all cylinders in four different resolutions. Data of coarse resolution from the third order expansions were not listed here. Along each row in the table for each cylinder, only relatively small differences are there. Table 7 shows the computed torque value about the axis of symmetry parallel to $x$. Consistent results are observed between different runs. 
Table 8 presents the wall clock time for the above four runs at different resolutions. Although the last case in the table used more elements than the rest three runs, the polynomial expansion in the third run is higher than the last one. On 576 elements at $6^{\text {th }}$ order expansion in all directions, the third run has more quadrature points and more degrees of freedom than the last run on 1,296 element. Therefore, the third run took longer time than the last one.

\subsection{Flow Past Five Hundred Randomly Arranged Cylinders}

To show the capability and efficiency of VCM in modeling flows with numerous cylinders, we present the last example with 500 cylinders between two parallel walls. Using dimensionless unit for the following discussion, the radii of these cylinders are $a=0.5$. Each wall measures from 0 to 2 in $x$ and from -16 to 16 in $z$. Between two walls at $y= \pm 16$, separately, there are 500 cylinders randomly arranged and parallel to $x$. A fully developed plane Poiseuille flow between two parallel walls is directed to these cylinders at the entrance plane $z=-16$. Therefore, the fluid boundary condition at the inlet is a parabolic profile with a peak value $w=10$ and zero at two walls where nonslip boundary conditions are specified. At the exit plane $z=16$, a Neumann boundary condition $\partial w / \partial z=0$ was imposed. Along the length of cylinders in $x$ direction, periodic boundary conditions were imposed at $x=0$ and 2 , respectively, to eliminate the end effect of cylinders. Both the density $\rho$ and dynamic viscosity $\mu$ of the fluid were scaled to 1 for simplicity.

Performing DNS for such a flow with 500 internal boundaries is prohibitively expensive, even if with our domain decomposition method and the parallel algorithm, and requires fully resolving all boundary layers. Therefore, only VCM runs were performed. The final computational mesh discretized the domain with only 32 hexahedral elements in both $y$ and $z$ and 1 element in $x$, a total of 1,024 elements. Within each element along each direction, polynomial expansions at $3^{\text {rd }}$ through $5^{\text {th }}$ orders were used in the computation.

Figure 11 presents the mainstream component of the fluid velocity in the plane perpendicular to both walls and a cross-sectional view of the random layout of cylinders which are marked with solid black circles in the figure for clarity. Because 500 cylinders displace significant amount of volume from the fluid between two parallel walls, the flow was accelerated. From the observation of this figure, based on the diameter of these cylinders, the peak Reynolds number in this flow is up to 40.

Figure 12 (a) presents the convergence history of the computed forces which were to counteract the lift and drag forces in the flow, using the penalty method described before, for cylinder No. 69, 119, and 374. The converged values of the balancing lift force have different signs due to their locations only. Comparing the magnitude of the balancing lift and drag, the latter is obviously larger since the main flow is aligned with the direction of drag. Figure 12 (b) presents the convergence history of the 


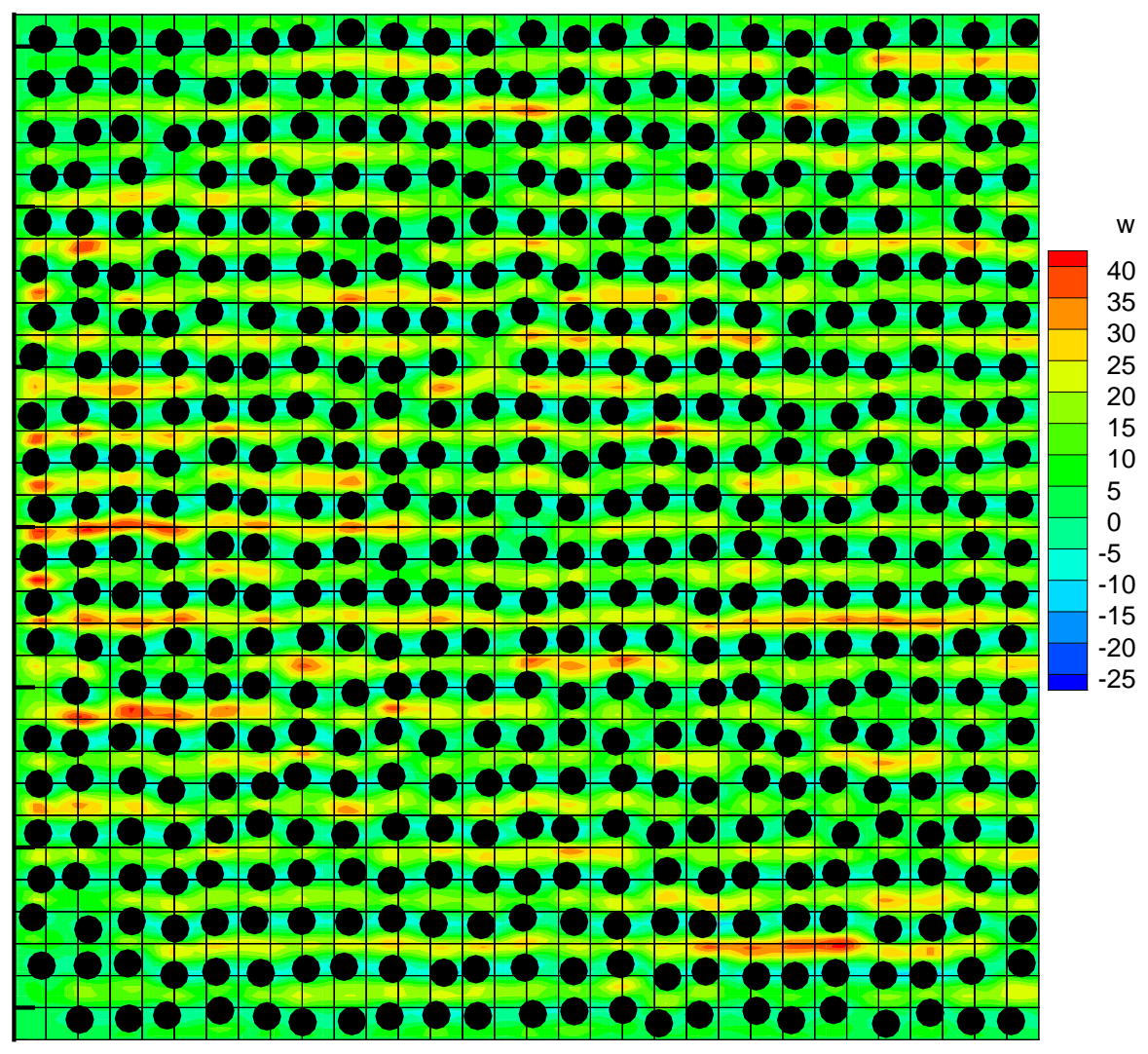

Fig. 11. Mainstream fluid velocity contour lines in the plane $x=1$ and the configuration of 500 cylinders from the VCM run on 1,024 elements at $5^{\text {th }}$ order expansion

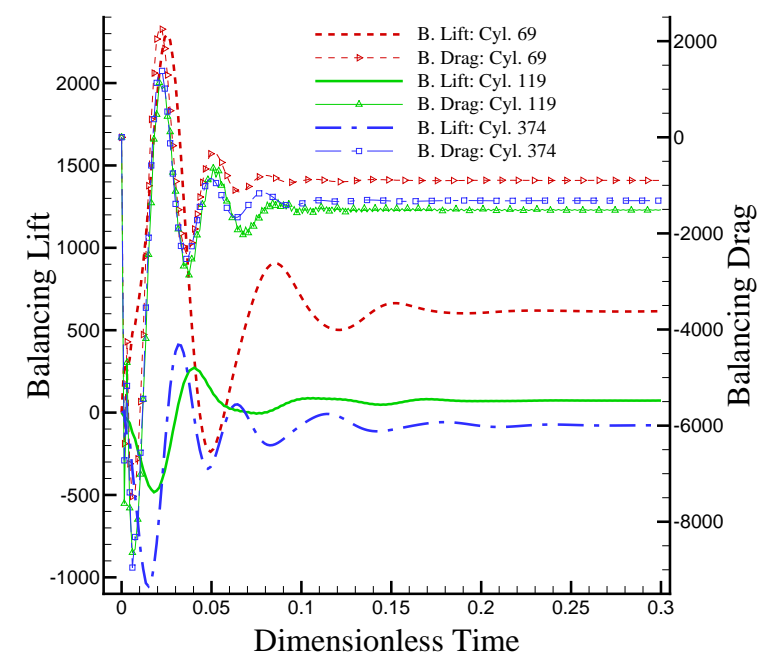

(a)

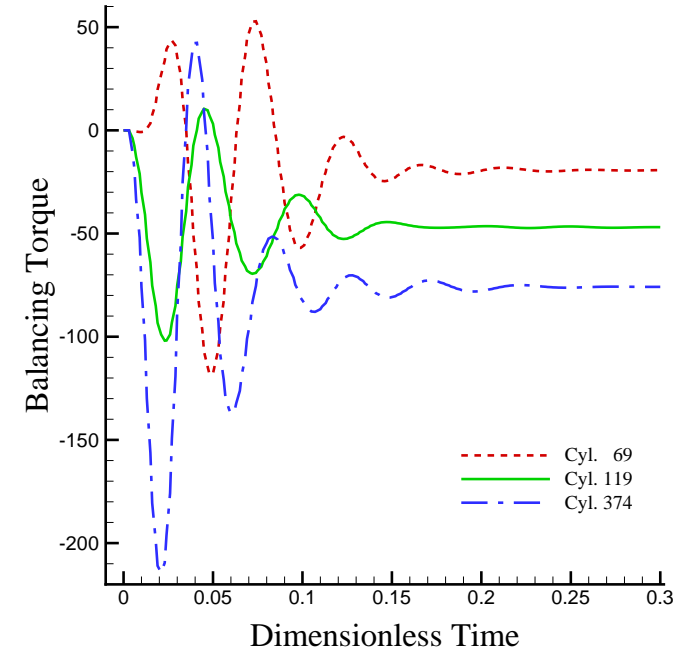

(b)

Fig. 12. Convergence of (a) the balancing lift and drag; (b) the balancing torque, for cylinders 69,119 , and 374 , in dimensionless units, from the VCM run on 1,024 elements at $5^{\text {th }}$ order expansion 
computed torque values to overcome the torques from the fluid on cylinder No. 69, 119 , and 374 . The converged torque on each cylinder is to offset the rotation if these cylinders were free to rotate in response to the shear stress and friction from the fluid.

\section{Conclusion}

This paper developed the Virtual Cylinders Model to investigate viscous and shear flows over vegetation stems, approximated as thin cylinders, growing in wetlands. This model possesses the abilities to accurately simulate an individual vegetation stem and to economically handle a bulk of numerous stems altogether. This model treats the fluid in an Eulerian reference and vegetation stems in Lagrangian references in order to fully utilize the efficiency of the Eulerian method and simplicity and accuracy of the Lagrangian method. The interaction between the fluid and the vegetation, i.e., the force and torque, are computed with the Lagrangian description and then added into the Eulerian solver for the flow field.

From the computational intensity point of view, this model simplifies a two-phase flow, fluid-vegetation interaction, into almost a single fluid phase flow. The avoidance of resolving the boundary layers around vegetation stems, especially when they move, is the key to its impressive economy in computational intensity. The accuracy of the VCM, especially in computing interaction forces and torques, was achieved owing to the exceptional spatial resolvability of the modal spectral element method in obtaining numerical solutions to the incompressible Navier-Stokes equations and numerical Gaussian quadrature. The capability of predicting drag forces without a body-fitting mesh was demonstrated in comparisons between VCM runs and DNS runs where good agreement was illustrated.

The VCM model possesses several advantages over other existing methods. First, it is perhaps the most economical method among other methods that use source terms to simulate the effect of cylinders. This is because only one kernel function is needed for each vegetation stem, compared to Immersed Boundary which uses numerous filter functions on all boundary grids and requires repetitive interpolations between fluid grids and solid boundary grids. This largely reduced the computational overhead in addition to the main flow field solver. Second, it has a wide range of applicability in terms of the Reynolds number, as the kernel function is adjustable for different flow conditions. Third, it is applicable to simulate vegetation stems with non-circular cross sections.

This paper has demonstrated the efficiency and accuracy of the VCM model for simulating the interaction flow with many vegetation cylinders of small diameters. Because of the unique and economical formulation of $\mathrm{VCM}$, it is unnecessary to resolve the boundary layer around each cylindrical surface. This provides a practical approach to modeling viscous flows interacting with numerous vegetation stems in 
a larger scale that other methods may become very slower. Potential applications of such a economical approach include simulations of wetland hydrodynamics, transport processes in ecological engineering, and fluid-structure interactions in the chemical and energy industries.

Subsequent work will investigate flows at higher Reynolds numbers over vegetation arrays of varied configuration and under medium shear rate. Meanwhile, studies will also be towards flow over deformable vegetation stems.

\section{Acknowledgements}

This research was supported by National Science Foundation (grants DMS-1115546, DMS-1318988, NSF/LEQSF(2010-13)-Track-2-LSU). The computational resources were provided by XSEDE (which is supported by National Science Foundation grant ACI-1053575), Louisiana Optical Network Initiative, and High Performance Computing at Louisiana State University.

\section{References}

[1] M. Fonseca, J. Fisher, J. Zieman, G. Thayer, Influence of the seagrass, Zostera marina, on current flow, Estuarine Coastal Shelf Science 15(4) (1982) 351-364.

[2] C. Peterson, R. Luettich, F. Micheli, G. Skilleter, Attenuation of water flow inside seagrass canopies of differing structure, Marine Ecology Progress Series 268 (2004) 8192 .

[3] M. Ghisalberti, H. Nepf, Mixing layers and coherent structures in vegetated aquatic flows, Journal of Geophysics Research 107(C2) (2002) 1-11.

[4] H. Nepf, Drag, turbulence and diffusion in flow through emergent vegetation, Water Resources Research 35(2) (1999) 479-489.

[5] L. Augustin, J. Irish, P. Lynett, Laboratory and numerical studies of wave damping by emergent and near-emergent wetland vegetation, Coastal Engineering 56(3) (2009) $332-340$.

[6] R. Jadhav, Q. Chen, J. Smith, Spectral distribution of wave energy dissipation by salt marsh vegetation, Coastal Engineering 77 (2013) 99-107.

[7] I. Möller, T. Spencer, J. French, D. Leggett, M. Dixon, Wave transformation over salt marshes: A field and numerical modeling study from North Norfolk, England, Estuarine, Coastal Shelf Science 49(3) (1999) 411-426.

[8] H. Coops, N. Geilen, H. Verheij, R. Boeters, G. Velde, Interactions between waves, bank erosion and emergent vegetation: an experimental study in a wave tank, Aquatic Botany 53(3-4) (1996) 187-198. 
[9] E. Gacia, C. Duarte, Sediment Retention by a Mediterranean Posidonia oceanica Meadow: The Balance between Deposition and Resuspension, Estuarine, Coastal Shelf Science 52(4) (2001) 505-514.

[10] F. Lopez, M. Garcia, Open-channel flow through simulated vegetation: Suspended sediment transport modeling, Water Resources Research 34(9) (1998) 2341-2352.

[11] L. Ward, W. Kemp, W. Boynton, The influence of waves and seagrass communities on suspended particulates in an estuarine embayment, Marine Geology 59(1-4) (1984) 85-103.

[12] M. Anderson, J. Smith, S. McKay, Wave Dissipation by Vegetation, Coastal and Hydraulics Engineering Technical Note ERDC/CHL CHETN-I-82.

[13] N. Loder, J. Irish, M. Cialone, T. Wamsley, Sensitivity of hurricane surge to morphological parameters of coastal wetlands, Estuarine, Coastal Shelf Science 84 (2009) 625-636.

[14] T. Wamsley, M. Cialone, J. Smith, B. Ebersole, A. Grzegorzewski, Influence of landscape restoration and degradation on storm surge and waves in southern Louisiana, Natural Hazards 51 (2009) 207-224.

[15] H. Zhao, Q. Chen, Modeling Attenuation of Storm Surge over Deformable Vegetation. Part I: Methodology and Verification, Journal of Engineering Mechanics 140(12), 04014090 (2014) 1-11.

[16] M. Zdravkovich, Flow around circular cylinders. Volume 2: Applications, Oxford University Press, 2003.

[17] C. Li, K. Yan, Numerical investigation of wave-current-vegetation interaction, Journal of Hydraulic Engineering 133(7) (2007) 794-803.

[18] V. Neary, Numerical solution of fully developed flow with vegetative resistance, Journal of Engineering Mechanics 129(5) (2003) 558-563.

[19] Y. Shimizu, T. Tsujimoto, Numerical analysis of turbulent open-channel flow over vegetation layer using a $\kappa-\epsilon$ turbulence model, Journal of Hydroscience and Hydraulic Engineering 11(2) (1994) 919-937.

[20] M.-L. Zhang, C. Li, Y.-M. Shen, A 3D non-linear $\kappa-\epsilon$ turbulent model for prediction of flow and mass transport in channel with vegetation, Applied Mathematical Modelling 34(4) (2010) 1021-1031.

[21] J. Cui, V. Neary, LES study of turbulent flows with submerged vegetation, Hydraulic Research 46(3) (2008) 307-316.

[22] C. Li, L. Yu, Hybrid LES/RANS modelling of free surface flow through vegetation, Computers \& Fluids 39 (2010) 1722-1723.

[23] X. Su, C. Li, Large eddy simulation of free surface turbulent flow in partly vegetated open channels, International Journal for Numerical Methods in Fluids 39(10) (2001) 919-937. 
[24] A. Chakrabarti, Q. Chen, H. Smith, D. Liu, Large eddy simulation of unidirectional and oscillatory flows through vegetation, Journal of Engineering Mechanics (2015) under review.

[25] Q. Chen, H. Zhao, Theoretical Models for Wave Energy Dissipation Caused by Vegetation, Journal of Engineering Mechanics 138(2) (2012) 221-229.

[26] C. Peskin, Flow patterns around heart valves: A digital computer method for solving the equations of motion, Ph.D. thesis, Albert Einstein College of Medicine, Yeshiva University (1972).

[27] C. Peskin, The fluid dynamics of heart valves: experimental, theoretical and computational methods, Annual Review of Fluid Mechanics 14 (1981) 235-259.

[28] S. Unverdi, G. Tryggvason, A front-tracking method for viscous, incompressible, multifluid flows, Journal of Computational Physics 123 (1992) 25-42.

[29] L. Fauci, A. McDonald, Sperm motility in the presence of boundaries, Bull. Math. Biol. 57 (1995) 679-699.

[30] E. Saiki, S. Biringen, Numerical Simulation of a Cylinder in Uniform Flow: Application of a Virtual Boundary Method, Journal of Computational Physics 123 (1996) 450-465.

[31] J. Mohd-Yosuf, Combined immersed boundary/b-spline methods for simulation of flow in complex geometries, Annu. Res. Briefs, Cent. Turbul. Res. 14 (1997) 317-328.

[32] R. Mittal, G. Iaccarino, Immersed boundary methods, Annual Review of Fluid Mechanics 37 (2005) 239-261.

[33] L. Leonard, M. Luther, Flow hydrodynamics in tidal marsh canopies, Limnology and Oceanography 40(8) (1995) 1474-1484.

[34] L. Leonard, D. Reed, Hydrodynamics and sediment transport through tidal marsh canopies, Journal of Coastal Research 36(2) (2002) 459-469.

[35] D. Liu, Q. Chen, Y. Wang, Spectral element modeling of sediment transport in shear flows, J. Computer Methods in Applied Mechanics and Engineering 200 (2011) 16911707 .

[36] D. Liu, Y. Lvov, W. Dai, Joint simulations of confined diffusion inside nanotubules, J. Computational and Theoretical Nanoscience 8(2) (2011) 1-11.

[37] S. Lomholt, M. Maxey, Force coupling method for particles sedimenting in a channel: Stokes flow, J. Comp. Phys. 184 (2003) 381-405.

[38] M. Maxey, B. Patel, Localized force representations for particles sedimenting in Stokes flow, Int. J. Multiphase Flow 27 (2001) 1603-1626.

[39] D. Liu, M. Maxey, G. Karniadakis, A fast method for particulate microflows, J. Microelectromechanical System 11 (2002) 691-702.

[40] D. Liu, M. Maxey, G. Karniadakis, Modeling and optimization of colloidal micro-pumps, J. Micromechanics and Microengineering 14 (2004) 567-575. 
[41] D. Liu, Spectral Element/Force Coupling Method: Application to Colloidal MicroDevices and Self-Assembly Structures in 3D Complex-Geometry Domains, Ph.D. thesis, Brown University (2004).

[42] S. Kim, S. Karrila, Microhydrodynamics Principles and Selected Applications, Dover Publications, Inc., 2005.

[43] G. Karniadakis, S. Sherwin, Spectral/hp Element Methods for Computational Fluid Dynamics, Oxford University Press, 2005.

[44] T. Warburton, S. Sherwin, G. Karniadakis, Spectral Basis Functions for 2D Hybrid hp Elements, SIAM Journal on Scientific Computation 20(5) (1999) 1671-1695.

[45] G. Karniadakis, M. Israeli, S. Orszag, High-order splitting methods for the incompressible Navier-Stokes equations, J. Comp. Phys. 97 (1991) 414-443. 